FONOS Y ESTRUCTURAS SILÁBICAS MÁS FRECUENTES EN EL

HABLA GLOSOLÁLICA (HABLAR EN LENGUAS) DE TRES HISPANOHABLANTES

Most frecuent phones and syllabic structures in glossalics (tongue speaking) of three spanish speakers

Gastón Felipe Salamanca Gutiérrez* Cristián Andrés Cabezas Mundaca**

Resumen

En esta presentación se determinan las unidades fónicas segmentales $-\mathrm{y}$ su frecuencia - presentes en el habla glosolálica de tres hispanohablantes, y se establece que en ella aplica la hipótesis de que el tipo silábico universal y más frecuente es CV (Obediente, 2007; Burquest, 2009). Para concretar estos objetivos se contabilizaron los segmentos y las estructuras silábicas presentes en 57 grupos fónicos, correspondientes a 2 minutos y 44 segundos de habla glosolálica. En consonancia con el diseño de investigación descrito, hemos dispuesto este trabajo como sigue: en primer lugar se presenta el marco de referencia en que se inscribe esta presentación; posteriormente se presentan los objetivos que se pretende alcanzar; en seguida, se presenta paso a paso la metodología adoptada; luego se presenta el análisis y los resultados obtenidos, con comentarios para cada clase natural; y, finalmente, a modo de conclusión, se presenta un resumen y discusión de los hallazgos, y posibles proyecciones del trabajo realizado.

Palabras clave: Glosolalia, Hablar en lenguas, Estructuras silábicas, Fonética segmental.

Abstract

This presentation will determine the segmental phone units -and their frecuencypresent in the glossalic speech of three Spanish speakers. It will also establish that in glossalic speech the hypothesis of the universal and most frecuent syllabic pattern CV can be applied (Obediente, 2007; Burquest, 2009). To accomplish the objectives, we counted the segments and syllabic structures occurring in 57 phonic groups corresponding to 2 minutes and 44 seconds of glossalic speech. In line with the described research design, we have ordered this work as follows: first, we present a frame of reference which fits this presentation; second, the objectives to be achieved; third, the step by step methodology adopted; then, the analysis and results, with comments for each natural class; and finally, as a conclusion, a summary and discussion of the discoveries, and the possible projections of this work.

Key words: Glossolalia, Speaking in tongues, Syllable structure, Segmental phonetics.

\title{
INTRODUCCIÓN
}

El término glosolalia proviene del griego glossa, que significa "lengua"; y laleín, que significa "hablar". También es conocida como el don de lenguas, 
expresión que el Diccionario de la Real Academia Española define como una "capacidad sobrenatural que permite a alguien hablar lenguas que desconoce, y en especial, la concedida por Dios a los apóstoles en Pentecostés".

Desde una perspectiva psicológica, en tanto, la glosolalia es definida por el mismo $D R A E$ como un "Lenguaje ininteligible, compuesto por palabras inventadas y secuencias rítmicas y repetitivas, propio del habla infantil, y también común en estados de trance o en ciertos cuadros psicopatológicos".

Desde una perspectiva lingüística, en tanto, Cardona la define como una:

[...]Verbalización automática sin contenido semántico; se manifiesta a través de secuencias de sonidos que poseen una semejanza aparente con el habla ordinaria pero que en realidad no son realizadas a partir de un código verdadero. Sin embargo, aunque incomprensibles en el plano formal, las producciones glosolálicas pueden transmitir "globalmente", de modo empático, un determinado contenido en presencia de interlocutores fuertemente motivados o en situaciones comunicativas favorables. Así se explica que se recurra con frecuencia a la glosolalia dentro de manifestaciones religiosas de grupos estrechamente unidos como los de las iglesias pentecostales (128).

Nuestra presentación se inscribe en este segundo ámbito y su objetivo es analizar, desde un punto de vista fonético-articulatorio, la manifestación de la glosolalia en tres hispanohablantes, reconocidos dentro de la religión evangélica. Específicamente, se pretende determinar las unidades fónicas segmentales $-\mathrm{y}$ su frecuencia - presentes en este tipo de habla, y determinar si aplica en él la hipótesis de que el tipo silábico universal y más frecuente es CV (Obediente, 2007; Burquest, 2009). Para concretar estos objetivos se transcriben fonéticamente 57 grupos fónicos y luego se contabilizan los segmentos y las estructuras silábicas.

En consonancia con el diseño de investigación descrito, hemos dispuesto este trabajo como sigue: en primer lugar se presenta el marco de referencia en que se inscribe esta presentación; posteriormente se presentan los objetivos generales y específicos que se pretende alcanzar; en seguida se presenta paso a paso la metodología adoptada; luego se presenta el análisis y los resultados obtenidos, con comentarios para cada clase natural; $y$, finalmente, a modo de conclusión, se presenta un resumen y discusión de los hallazgos, una evaluación respecto de la consecución o no de los objetivos, y posibles proyecciones del trabajo realizado.

\section{MARCO DE REFERENCIA}

\subsection{El segmento y la sílaba}

Este artículo se inscribe dentro de la fonética articulatoria descriptiva, como se presenta, entre otros muchos autores, en Quilis y Fernández (1973), Martínez-Celdrán (1993, 1996), Obediente (2007) y Burquest (2009). Uno de los aspectos que se

\footnotetext{
${ }^{1}$ Utilizamos aquí la versión on line disponible en http://www.rae.es/rae.html 
enfatiza en esta disciplina es que los sonidos del habla se agrupan formando una unidad mayor: la sílaba (Martínez-Celdrán, El sonido, 19).

Para la determinación de las fronteras de la sílaba se puede considerar un criterio sustancial (basado en la articulación y/o hechos acústicos) y un criterio funcional (división fundada en la posibilidad de agrupamiento de los fonemas, su susceptibilidad de, por sí solos, formar sílabas). De acuerdo con este último criterio, una misma secuencia puede ser interpretada como una sola unidad, o como dos, dependiendo de la lengua de que se trate. Es el caso, por ejemplo, de la secuencia "mb", que se interpreta como dos unidades en una lengua como el español, pero como un solo fonema oclusivo prenasalizado en algunas lenguas africanas (Obediente, 179).

La teoría fonológica contemporánea ha propuesto que los segmentos fonológicos no se distribuyen en la sílaba al azar, sino que tienden a agruparse de acuerdo con su fuerza consonántica o grado de consonantalidad (Hooper, 206). Algunas conclusiones que, de acuerdo con Burquest (158), se pueden obtener de este hecho son las siguientes:

1. Las semivocales son las consonantes más débiles (o más sonoras). Prácticamente son idénticas a las vocales fonéticamente hablando, y por lo tanto puede ser muy probable que se den hacia la parte nuclear de la sílaba.

2. Las líquidas y nasales son las que siguen en debilidad. En algunas lenguas y en algunos ambientes se vuelven silábicas (es decir, aparecen como la cima de la sílaba), especialmente si no hay ninguna vocal adyacente.

3. Las obstruyentes (plosivas, africadas y fricativas) son las menos parecidas a las vocales. Raras veces funcionan en la cima silábica, por lo tanto son las más fuertes de las consonantes.

La estructura silábica se ha representado tradicionalmente como:

$\sigma$

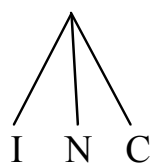

Esto es, un inicio, un núcleo y una coda en un mismo nivel jerárquico. Sin embargo, hoy se prefiere una estructura diferente:

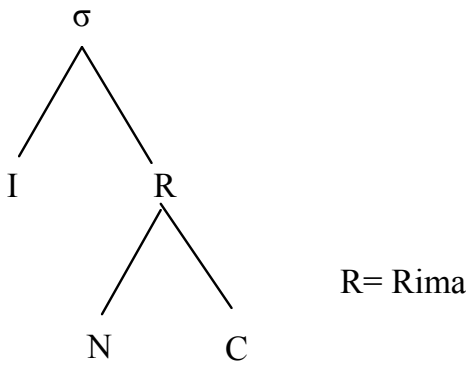


Esta estructura refleja que la coda está en un nivel jerárquico inferior al inicio, lo que tiene dos fundamentos sólidos (Burquest, 160): a) La sílaba CV (esto es, una sílaba sin coda) es considerada la sílaba universal, tanto desde el punto de vista de la adquisición del lenguaje como desde el punto de vista tipológico, y b) en las lenguas, en general, en la posición de inicio, ocurren más fonemas $-\mathrm{y}$ con mayor distintividad - que en la posición de coda.

Así, por ejemplo, siguiendo estos principios, el autor segmenta la secuencia (/pasuNnum/) del aguaruna ${ }^{2}$ de la siguiente manera:

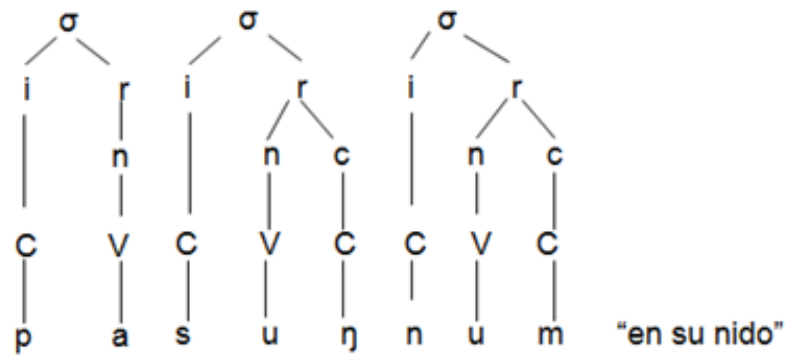

Esta segmentación asume que generalmente se da el caso que los cortes silábicos se encuentran al interior de la palabra entre una vocal y una consonante que le sigue, la que también tiene una vocal que le sigue, es decir, V.CV. Por consiguiente, el primer corte silábico de la palabra se encuentra entre la [a] y la [s] (Burquest, 163).

En este contexto, la afirmación de Obediente (182) en cuanto a que "La única sílaba universal (existente en todas las lenguas) es [...] la de tipo CV; los otros tipos de sílaba varían según las lenguas" es central para nuestro trabajo.

\subsection{El hablar en lenguas}

Crystal (265) destaca dos aspectos centrales presentes en la glosolalia: a) desde una perspectiva sociolingüística, tiene como función única actuar como signo de las creencias del hablante - o como muestra de conversión-, pero carece de referencia convencional; y b) su estructura lingüística formal es muy distinta de las lenguas comunes, más simple y más reiterativa (a pesar de las afirmaciones del hablante de que está articulando una lengua real pero desconocida).

Por otra parte, la atención prestada a este tipo de "lenguaje" tiene, aunque escasa, una tradición. Prueba de ello son, entre otros, los trabajos de Samarin (Variation and variables) y Jakobson y Waugh (203-208). Estos últimos autores definen este tipo de habla como un tipo de actividad creadora verbal o cuasiverbal que se caracteriza por el uso de los sonidos del habla con privación de la función discriminadora de sentido, que es dirigido a un público humano real o con la intención de que sea recepcionado por un espíritu divino (Jakobson y Waugh, 203).

2 Una de las lenguas habladas en el Perú. 
En este mismo trabajo dan cuenta de dos reportes de Samarin (Tongues of Men y Variation and variables) en los que se mencionan casos de glosolalia. En el primer trabajo se informa de un rezo de un ministro presbiteriano que consta de 28 "grupos de respiración", donde se presentan cuarenta grupos de $n d r$ y treinta $n t r$, más once de $n t$ y $n d$. El rezo en cuestión comienza con la oración o grupo de respiración kupóy shăndré filé sundrukumă shăndré lása hóya tăkí, y está construido con grupos similares. En el segundo trabajo se contienen dos breves muestras de discurso glosolálico neopentecostal, una de 43 y otra de 38 segundos, en donde se incluyen quince combinaciones de $n d$ cada una. En estos dos casos destaca la preferencia por las combinaciones con $/ \mathrm{n} / \mathrm{más} / \mathrm{t}$, $\mathrm{d} /$, las que podrían interpretarse como oclusivas prenasalizadas (Trubetzkoy, 169).

Además, es interesante la observación que hacen Jakobson y Waugh respecto de la necesidad de recopilación de glosolalia proveniente de distintas latitudes (a lo que nuestra investigación, por cierto, contribuye):

Antes que permitir algunas inferencias generalizadas, tales ejemplos de correspondencias interculturales en el arte verbal glosolálico, y podría añadirse también "supraconsciente", requieren una recopilación de registro de glosolalia internacional o interdenominativa completa, en el sentido más amplio de aquel término griego [...] La dependencia de los textos glosolálicos del esquema sonoro de la lengua nativa del emitente así como de las variaciones individuales en la conformación y distribución de grupos sonoros repetidos concurre con los principios ubicuos comunes de la estructuración de composiciones esotéricas medio improvisadas y medio tradicionales con cuasipalabras. (205-206).

\section{OBJETIVOS}

a) Determinar los fonos y estructuras silábicas más frecuentes en el habla glosolálica de tres hispanohablantes.

b) Establecer si la hipótesis CV aplica al habla glosolálica.

\section{METODOLOGÍA}

\subsection{Los datos}

\subsubsection{Recolección del material}

Se obtuvieron archivos en formato de audio mp3, de sermones de pastores evangélicos donde se manifestaba la glosolalia ${ }^{3}$.

Del pastor Y. Á., se seleccionaron 3 sermones: "El rapto", "La desobediencia" y "Las diez vírgenes". En ellos se encontraron 8, 4, y 7 grupos fónicos con manifestación de glosolalia, correspondientes a 12,9, y 12 segundos, respectivamente.

3 Estos datos nos fueron facilitados por el Ministerio Evangelístico Águilas de Jesús; específicamente, por el pastor Gustavo Torres. Agradecemos esta muestra de confianza en un tema tan sensible dentro del mundo protestante. 
Del pastor D. G. se seleccionó el sermón "Gacelas y Leones". En él se encontraron 15 grupos fónicos con manifestación de glosolalia, correspondientes a 28 segundos.

Del pastor E. B. se seleccionó un sermón sin título, en el que se encontraron 24 grupos fónicos con manifestación de glosolalia, correspondientes a 1 minuto y 43 segundos.

Así, en total se analizaron 58 grupos fónicos, correspondientes a 2 minutos y 44 segundos.

\subsubsection{Transcripción de los segmentos}

Para reproducir los enunciados a una velocidad lenta, y así optimizar la discriminación de algunos sonidos, utilizamos el programa Audacity y el reproductor de Windows Media. Para la transcripción fonética se utilizó el Alfabeto Fonético Internacional.

\section{ANÁLISIS Y RESULTADOS}

Presentamos a continuación las Tablas con la cuantificación de los segmentos y estructuras silábicas, y los comentarios que emergen a partir de ellas. Para la comparación de nuestros datos con las tendencias de las lenguas en general, hemos seguido de cerca la información contenida en la sección "Universales fonológicos: Sistemas vocálicos y consonánticos", de Burquest (2009: 47-53).

\subsection{Segmentos y Diptongos}

\subsubsection{Segmentos} siguiente:

Los segmentos detectados y su frecuencia de aparición, respecto del total es el

Tabla 1. Segmentos vocálicos y consonánticos dispuestos en orden decreciente de frecuencia

\begin{tabular}{|c|c|c|}
\hline Fonos vocales & $\mathbf{n}$ & $\mathbf{\%}$ \\
\hline$[\mathrm{a}]$ & 355 & 28,3 \\
\hline$[\mathrm{o}]$ & 111 & 8,9 \\
\hline$[\mathrm{e}]$ & 87 & 6,9 \\
\hline$[\mathrm{i}]$ & 60 & 4,8 \\
\hline$[\mathrm{u}]$ & 11 & 0,9 \\
\hline$[\leftrightarrow]$ & 2 & 0,2 \\
\hline Total vocales & 626 & 50 \\
\hline Consonantes & $\mathrm{n}$ & $\%$ \\
\hline$[\mathrm{s}]$ & 85 & 6,8 \\
\hline$[\mathrm{b}]$ & 75 & 5,9 \\
\hline$[\mathrm{P}]$ & 68 & 5,4 \\
\hline$[\mathrm{m}]$ & 54 & 4,3 \\
\hline$[\mathrm{n}]$ & 39 & 3,1 \\
\hline$[\mathrm{\rho}]$ & 38 & 3,1 \\
\hline
\end{tabular}




\begin{tabular}{|c|c|c|}
\hline$[\Sigma]$ & 37 & 3,0 \\
\hline$[\mathrm{t}]$ & 35 & 2,8 \\
\hline$[\mathrm{k}]$ & 34 & 2,7 \\
\hline$[\mathrm{d}]$ & 33 & 2,6 \\
\hline$[\mathrm{h}]$ & 33 & 2,6 \\
\hline$[\mathrm{l}]$ & 21 & 1,7 \\
\hline$[\varphi]$ & 18 & 1,4 \\
\hline$[\mathrm{B} 4]$ & 16 & 1,3 \\
\hline$[\mathrm{p}]$ & 14 & 1,1 \\
\hline$[\mathrm{V}]$ & 14 & 1,1 \\
\hline$[\mathrm{z}]$ & 4 & 0,3 \\
\hline$[\Delta 4]$ & 3 & 0,2 \\
\hline$[\mathrm{kh}]$ & 3 & 0,2 \\
\hline$[\times]$ & 2 & 0,1 \\
\hline$[\otimes 4]$ & 1 & 0,1 \\
\hline$[\mathrm{ts}]:$ & 1 & 0,1 \\
\hline Total consonantes & 628 & 50 \\
\hline Total general & 1254 & 100 \\
\hline
\end{tabular}

\subsubsection{Vocales}

Tabla 2. Fonos vocálicos y su frecuencia

\begin{tabular}{|c|c|c|}
\hline Fonos vocálicos & $\mathbf{n}$ & $\mathbf{\%}$ \\
\hline$[\mathrm{a}]$ & 355 & 56,7 \\
\hline$[\mathrm{o}]$ & 111 & 17,7 \\
\hline$[\mathrm{e}]$ & 87 & 13,9 \\
\hline$[\mathrm{i}]$ & 60 & 9,6 \\
\hline$[\mathrm{u}]$ & 11 & 1,8 \\
\hline$[\leftrightarrow]$ & 2 & 0,3 \\
\hline Total vocales & $\mathbf{6 2 6}$ & $\mathbf{1 0 0}$ \\
\hline
\end{tabular}

\section{Observaciones}

- Como se observa, en nuestro corpus ocurren 6 segmentos vocálicos. Es un sistema simétrico, pues los sonidos están repartidos en forma equitativa en el espacio articulatorio disponible. En este aspecto, lo que se presenta en el habla glosolálica que nos ocupa es compatible con la tendencia de los sistemas fonético-fonológico de las lenguas en general (Burquest, 4).

- En cuanto a la frecuencia de las vocales, se observa que $[\alpha]$ y $[\leftrightarrow]$ son las vocales más y menos frecuentes, respectivamente. La alta frecuencia de $[\alpha]$ es compatible con la tendencia de las lenguas en general, pues en la gran mayoría de ellas, acaso en todas, se presenta y con una alta frecuencia.

\subsubsection{Consonantes}




\subsection{Oclusivas}

Tabla 3. Fonos consonánticos oclusivos y su frecuencia.

\begin{tabular}{|c|c|c|}
\hline Fonos oclusivos & $\mathbf{n}$ & \% \\
\hline$[\mathrm{b}]$ & 75 & 38,7 \\
\hline$[\mathrm{t}]$ & 35 & 18,0 \\
\hline$[\mathrm{k}]$ & 34 & 17,5 \\
\hline$[\mathrm{d}]$ & 33 & 17,0 \\
\hline$[\mathrm{p}]$ & 14 & 7,2 \\
\hline$[\mathrm{kh}]$ & 3 & 1,5 \\
\hline Total oclusivas & $\mathbf{1 9 4}$ & $\mathbf{1 0 0}$ \\
\hline
\end{tabular}

Observaciones

- Destaca, en primer lugar, la ocurrencia de una consonante compleja aspirada, aunque de baja frecuencia. Es probable que esta ocurrencia se deba al componente enfático que caracteriza a este tipo de habla (al menos en los casos estudiados).

- Por otro lado, en el punto de articulación bilabial predomina la consonante oclusiva sonora [ $\beta]$, ello se condice con la tendencia de las lenguas en general (Burquest, 51). Destaca, además, que $[\beta]$ ocurra prácticamente en todos los casos $(73$ de 75) en un contexto intervocálico (ver Anexo), contexto en el que no ocurre en español. Esto, al igual que la presencia de [kh], podría atribuirse a la fonización de un "contenido" enfático que este tipo de habla releva.

- Respecto de la zona alveolar, la cantidad similar de realizaciones de [t] y [d] no permite hacer afirmaciones categóricas. Sin embargo, destaca también la ocurrencia de la consonante alveolar sonora $[\delta] \square$ en posición intervocálica (ver Anexo), por lo que aplica aquí el último comentario que hemos hecho respecto [b].

- En la zona velar, por último, se releva el predominio absoluto de la consonante áfona $[\mathrm{k}]$, lo que también es compatible con la tendencia que presentan las

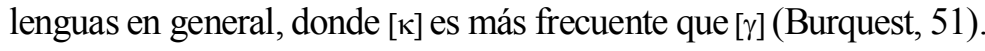

\subsection{Fricativas}

Tabla 4. Fonos consonánticos fricativos y su frecuencia.

\begin{tabular}{|c|c|c|}
\hline Fonos fricativos & $\mathbf{n}$ & $\mathbf{\%}$ \\
\hline$[\mathrm{s}]$ & 85 & 49,1 \\
\hline$[\Sigma]$ & 37 & 21,4 \\
\hline$[\mathrm{h}]$ & 33 & 19,1 \\
\hline$[\mathrm{v}]$ & 14 & 8,1 \\
\hline$[\mathrm{z}]$ & 4 & 2,3 \\
\hline Total fricativas & $\mathbf{1 7 3}$ & $\mathbf{1 0 0}$ \\
\hline
\end{tabular}

Observaciones

- Destaca, en primer lugar, la alta frecuencia de las consonantes fricativas (representan el 27,6\% del total de consonantes). Probablemente, la ocurrencia prominente del rasgo estridencia en las más frecuentes sea decisivo en este caso, pues también tributaría al aspecto enfático que este tipo de habla releva (al menos en los 
casos estudiados).

- En esta misma dirección, destaca también la altísima frecuencia de las fricativas sibilantes (representan el 71,1\% del total de consonantes fricativas).

- De acuerdo con Burquest (51), en las lenguas en general es más frecuente que la zona labiodental sea ocupada por la consonante sonora [v], constatación que se aviene con nuestros datos; en estos, incluso, $[\phi]$ no ocurre.

- La escasa ocurrencia de [z] se condice también con la tendencia en las lenguas en general (Burquest, 51).

- Es interesante observar que en la zona velar no ocurre ninguna consonante fricativa. De hecho, en la zona posterior en general -esto es, incluidas las zonas velar y glotal- se presenta solo un fono consonántico con este modo de articulación ([h]), el que, sin embargo, tiene una alta frecuencia. Esta constatación es interesante porque coincide con lo que se observa en lenguas con sistemas fonológicos muy pequeños, como el pirahá o el hawayano, en donde la clase natural que nos ocupa solo presenta los segmentos "s" y " $\mathrm{h}$ " (a lo menos como fonemas). Probablemente, el escaso esfuerzo articulatorio implicado en la producción de " $h$ " explique esta coincidencia.

\subsection{Aproximantes}

Tabla 5. Fonos consonánticos aproximantes y su frecuencia

\begin{tabular}{|c|c|c|}
\hline Fonos aproximantes & $\mathbf{n}$ & $\mathbf{\%}$ \\
\hline$[\varphi]$ & 18 & 47,4 \\
\hline$[\mathrm{B} 4]$ & 16 & 42,1 \\
\hline$[\Delta 4]$ & 3 & 7,9 \\
\hline$[\otimes 4]$ & 1 & 2,6 \\
\hline Total aproximantes & $\mathbf{3 8}$ & $\mathbf{1 0 0}$ \\
\hline
\end{tabular}

Observaciones

- Las consonantes aproximantes, en general, presentan una baja frecuencia (solo representan el 6,1\% del total de consonantes). Una posible explicación para la baja frecuencia de estos segmentos es que son contoides que no presentan un componente de estridencia/explosión y, por lo tanto, no tributan al efecto enfático que, como hemos dicho, este tipo de habla releva (al menos, en los casos estudiados).

- Si bien estos segmentos tienen, en general, una baja frecuencia, es interesante observar que la aproximante palatal $[\varphi]$ presenta 18 realizaciones, lo que constituye una cantidad relativamente importante. En este mismo contexto, es interesante observar que en la zona palatal solo ocurren 2 fonos: el que nos ocupa y la consonante lateral $[\times]$ (aunque con solo dos realizaciones). Al parecer, la preferencia por $[\varphi]$ para ocurrir en esta zona de articulación, igual que en el caso de [h], se debe al escaso esfuerzo articulatorio que requiere su realización.

\subsection{Africadas}

Tabla 6. Fonos africados y su frecuencia. 


\begin{tabular}{|c|c|c|}
\hline Fonos africados & $\mathbf{n}$ & $\mathbf{\%}$ \\
\hline [ts] & 1 & 100 \\
\hline Total africadas & $\mathbf{1}$ & $\mathbf{1 0 0}$ \\
\hline
\end{tabular}

Observaciones

- Es interesante observar que se produce solo un sonido africado y con solo una realización. La razón, creemos, se relaciona con lo que hemos venido comentando en algunos puntos anteriores. En efecto, en las consonantes africadas se atenúa la explosión propia de las consonantes oclusivas y la turbulencia/ruido característico de las fricativas, $y$, con ello, el efecto enfático que el habla glosolálica releva (al menos en los casos estudiados).

\subsection{Nasales}

Tabla 7. Fonos consonánticos nasales y su frecuencia.

\begin{tabular}{|c|c|c|}
\hline Fonos nasales & $\mathbf{n}$ & $\mathbf{\%}$ \\
\hline$[\mathrm{m}]$ & 54 & 58,1 \\
\hline$[\mathrm{n}]$ & 39 & 41,9 \\
\hline Total nasales & $\mathbf{9 3}$ & $\mathbf{1 0 0}$ \\
\hline
\end{tabular}

Observaciones

- Destaca la ocurrencia de las consonantes nasal bilabial y alveolar. Esta es la tendencia también en las lenguas en general (Burquest, 52).

4.1.1.2.6. Laterales

Tabla 8. Fonos consonánticos laterales y su frecuencia.

\begin{tabular}{|c|c|c|}
\hline Fonos laterales & $\mathbf{n}$ & $\mathbf{\%}$ \\
\hline$[l]$ & 21 & 91,3 \\
\hline$[\times]$ & 2 & 8,7 \\
\hline Total laterales & $\mathbf{2 3}$ & $\mathbf{1 0 0}$ \\
\hline
\end{tabular}

Observaciones

- Destaca la alta frecuencia de la consonante lateral alveolar, y la ocurrencia de una lateral palatal, aunque de muy baja ocurrencia. La mayor frecuencia de la lateral alveolar es también compatible con la tendencia de las lenguas en que estos segmentos se presentan.

\subsection{Vibrantes}

Tabla 9. Fonos consonánticos vibrantes y su frecuencia

\begin{tabular}{|c|c|c|}
\hline Fonos vibrantes & $\mathbf{n}$ & $\mathbf{\%}$ \\
\hline$[\mathrm{P}]$ & 68 & 64,2 \\
\hline$[\rho]$ & 38 & 35,8 \\
\hline Total vibrantes & $\mathbf{1 0 6}$ & $\mathbf{1 0 0}$ \\
\hline
\end{tabular}

Observaciones

- Destaca la ocurrencia de dos vibrantes. Es probable que la frecuencia relativamente importante de la vibrante múltiple, no habitual en las lenguas en 
general, se vincule con el componente enfático del habla glosolálica al que hemos hecho referencia en observaciones precedentes.

\subsubsection{Diptongos}

Tabla 10. Diptongos y su frecuencia.

\begin{tabular}{|c|c|c|}
\hline Diptongos & $\mathbf{n}$ & $\mathbf{\%}$ \\
\hline$[\alpha 19]$ & 33 & 73,3 \\
\hline$[\varepsilon 19]$ & 12 & 26,7 \\
\hline Total diptongos & $\mathbf{4 5}$ & $\mathbf{1 0 0}$ \\
\hline
\end{tabular}

Observaciones

- Es interesante observar que no se producen diptongos con la semivocal [v9].

- Por otro lado, se observa que ambos diptongos son decrecientes y que no participan las vocales posteriores plenas [o] ni [v].

- El hecho de que el diptongo formado con la vocal $[\alpha]$ sea el más frecuente se condice con la tendencia de las lenguas en general.

\subsection{Estructuras silábicas}

Las estructuras silábicas y sus respectivas frecuencias, en orden decreciente, son las que siguen:

Tabla 11. Estructuras silábicas y sus respectivas frecuencias.

\begin{tabular}{|c|c|c|}
\hline Estructura silábica & $\mathbf{n}$ & $\mathbf{\%}$ \\
\hline CV & 498 & 77,3 \\
\hline V & 42 & 6,5 \\
\hline CVC & 28 & 4,3 \\
\hline CD & 27 & 4,2 \\
\hline CCV & 15 & 2,3 \\
\hline D & 13 & 2,0 \\
\hline CCVC & 7 & 1,1 \\
\hline CDC & 7 & 1,1 \\
\hline VC & 5 & 0,8 \\
\hline CCDC & 1 & 0,2 \\
\hline CCD & 1 & 0,2 \\
\hline Total sílabas & $\mathbf{6 4 4}$ & $\mathbf{1 0 0}$ \\
\hline
\end{tabular}

Observaciones

- Destaca la ocurrencia de una variedad de tipos silábicos, aunque aquellos que tienen premargen complejo (CC) tienden a ser los menos frecuentes (y solo ocurren en el hablante E.B.).

- Dos consonantes pueden ocurrir como premargen, pero no como postmargen.

- La estructura silábica más frecuente es CV (77\%), con lo que, de acuerdo con nuestros datos, la glosolalia sigue la tendencia de las lenguas en general. Esta 
constatación es particularmente relevante porque responde a uno de los objetivos de nuestra investigación.

\section{CONCLUSIONES}

En relación con los análisis realizados y los resultados obtenidos, nos interesa relevar lo siguiente:

1. Respecto de los segmentos y sus respectivas frecuencias:

a) El sistema fonético segmental presente en nuestros datos está constituido por 6 sonidos vocálicos, 22 consonánticos y 2 diptongos.

Si bien el sistema vocálico de seis unidades --como el que nos ocupa-- no constituye la tendencia mayoritaria en las lenguas en general (Burquest, 2009: 4-6), sí cumple con el requisito de tendencia a la simetría que se ha reportado en ellas y que se sustenta en el principio de que el espacio acústico/articulatorio es utilizado en forma óptima.

Por otro lado, la alta frecuencia de $[\alpha]$ se condice con lo que ocurre en las lenguas en general.

Referente al inventario de consonantes, como hemos visto, se produce una sintonía entre nuestros resultados y lo que se describe como tendencia de las lenguas en general. En efecto, Burquest (50) — siguiendo a Crothers (1978), entre otrosseñala que:

Se espera que todas las lenguas tengan por lo menos los siguientes fonemas consonánticos: (1) plosivas sordas, (2) nasales, (3) una fricativa acanalada como $/ \mathrm{s} /$, (4) una consonante laríngea, usualmente $/ \mathrm{h} /$.

Todo lo anterior se condice con nuestros hallazgos en el habla glosolálica.

En términos más específicos, nuestros datos apoyan plenamente las siguientes observaciones de Burquest (51-52):

[...] para las plosivas sordas, generalmente se mantiene la jerarquía $/ \mathrm{p} /</ \mathrm{t} /<$ $/ \mathrm{k} /$; y para las plosivas sonoras generalmente se mantiene la jerarquía opuesta $/ \mathrm{b} />/ \mathrm{d} />/ \mathrm{g} /$. Lo que esto quiere decir es que si una lengua presenta un vacío en su serie de plosivas sordas, es más probable que falte la $/ \mathrm{p} /$; mientras que si existe un vacío en su serie de plosivas sonoras, es más probable que falte la /g/.[...] Así, para las fricativas sordas, es más probable que las labiales estén ausentes, y para la serie de las sonoras es más probable que la velar esté ausente si existen asimetrías en el sistema [...] Casi todas las lenguas tienen consonantes nasales. [...] si existen vacíos en la serie de consonantes nasales, es más probable que sea la nasal velar $/ \mathrm{N} /$ y otros lugares de articulación no anteriores los que falten.

b) En consonancia con lo señalado, resulta interesante observar que las consonantes 
oclusivas y fricativas ocurren con una alta frecuencia. Pensamos que esto no solo se debe a una tendencia de las lenguas en general, sino que, al menos en los casos que nos ocupan, habría una razón pragmática concomitante: el relevamiento de un aspecto enfático. En efecto, los enunciados analizados muestran un marcado componente de vehemencia, el que se expresa, en el plano fónico, en la intensidad de la voz, alargamiento vocálico, etc.; pero también mediante segmentos que, por sus rasgos intrínsecos, pueden manifestarlo de manera prominente (los sonidos oclusivos y fricativos). Habría aquí, en consecuencia, una fonización, con segmentos $a d$ hoc de un contenido pragmático específico. Puesto en otras palabras: a causa de que en el habla glosolálica estudiada la devoción vehemente no se puede expresar, por definición, por medio de secuencias inteligibles, esta se comunica, entre otros mecanismos, con la explosión oclusiva y la estridencia fricativa. Esta fonización segmental de la devoción vehemente explicaría, también: 1) la presencia de una consonante oclusiva aspirada $/ \mathrm{kn} /$, así como la alta frecuencia de una consonante vibrante múltiple $/ \rho /$ (ambas consonantes de escasa ocurrencia en las lenguas en general); 2) la ocurrencia de las consonantes oclusivas bilabial $[\beta]$ y alveolar $[\delta]$ en un contexto donde no ocurren en español ${ }^{4}$ (entre vocales); y 3) la baja frecuencia de las consonantes aproximantes y africadas.

Ahora bien, es importante subrayar que este aspecto enfático es relavado en los casos que nos ocupan, pero no se puede descartar que en otras muestras de habla glosolálica dicho aspecto pragmático no se releve en la misma medida. Sin ir más lejos, en los casos que hemos examinado se observan algunos matices, pues dicho componente es muy prominente en Y. Á. y D.G., pero se releva en menor medida en E.B. Así, a la luz de nuestros datos, una tipología del habla glosolálica debiera incluir el componente enfático como uno de los factores que puede caracterizar algunos casos, pero no podríamos afirmar que constituye un rasgo definitorio de todos y cada uno de ellos ${ }^{5}$.

En esta misma dirección, y como hemos dicho, E.B. es quien se distancia en mayor medida de la relativa homogeneidad que presenta el habla de los otros dos informantes, aunque no solo por el carácter menos vehemente de su "discurso", sino, también, por el hecho de que, en el plano segmental, su habla presenta los dos

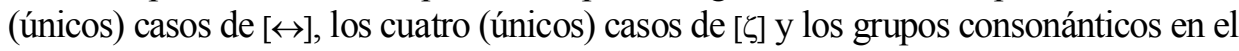
premargen silábico. Por lo mismo, es dable pensar que en este caso puede haber más de una lengua de sustrato, pero no contamos con el dato definitivo.

2. Respecto de la estructura silábica:

Las estructuras silábicas presentes en los casos de habla glosolálica

\footnotetext{
${ }^{4}$ Esta es la lengua de "sustrato" de los hablantes.

${ }^{5}$ Quienes escribimos este trabajo hemos podido apreciar, de hecho, que en algunos casos el habla glosolálica presenta rasgos suprasegmentales que evocan más bien una conversación y no una invocación vehemente.
} 
estudiados también se avienen con lo que se ha descrito como tendencia de las lenguas en general, pues CV es, por lejos, el patrón más recurrente $(77,3 \%)$. Esta constatación nos permite responder una de las preguntas de investigación centrales de nuestro trabajo.

Así, entonces, al reunir lo que se expresa en los puntos anteriores, y en un plano más especulativo, podemos afirmar que en el habla glosolálica investigada ocurre lo más representativo - en términos de tipos de segmentos, frecuencia y fonotaxis - de las tendencias que se presentan en las lenguas en general. En un grado no menor, también, estos fonos y secuencias silábicas son compatibles con los que se presentan en la lengua de sustrato de los sujetos (el español). Ahora, la presencia de sonidos que no están en el español — junto con la ocurrencia de algunos de ellos que sí están en su inventario pero que ocurren en contextos atípicos - se puede explicar, a lo menos en los casos estudiados, por el relevamiento de un aspecto pragmático: el imperativo de comunicar una devoción intensa, enfática, lo que, por las características del habla glosolálica, no se puede conseguir mediante los contenidos semánticos, pues estos son, por definición, ininteligibles.

Finalmente, algunas proyecciones que surgen de este trabajo son: a) determinar si en los datos analizados es recurrente la presencia de los grupos consonánticos "nd" y "nt", lo que, como señalamos en 1.2., es el caso en el habla glosolálica de un sujeto neopentecostal de USA; b) comparar estos datos con los aspectos más prominentes de la(s) lengua(s) de sustrato de los hablantes cuya habla se investigó (tarea no exenta de complicaciones, pues hay que tener presente que la comparación sería entre fonos, en el caso del habla glosolálica, y fonemas, en el caso de la lengua de sustrato); y, por supuesto, c) ampliar la muestra para determinar el alcance de las conclusiones e hipótesis explicativas que hemos planteado aquí.

\author{
Universidad de Concepción* \\ Facultad de Humanidades y Arte. Departamento de Español \\ Casilla 160-Correo, Concepción (Chile) \\ gaston.salamanca@gmail.com \\ Universidad de Concepción** \\ Calle Mariñán \#258 (interior) Cañete (Chile) \\ cristian.cabezas.m@gmail.com
}

OBRAS CITADAS

Burquest, Donald. Análisis fonológico: Un enfoque funcional. Dallas: SIL Interna- 
cional. 2009. Utilizamos versión on line disponible en:

http://www.sil.org/silepubs/Pubs/52264/Burquest_AnalisisFonologico_52264.pdf

Cardona, Giorgio. Diccionario de lingüística. Barcelona: Ariel, 1991.

Crothers, John. "Typology and universals of vowel systems". En: Greenberg, Joseph (Ed.). Universals of human language 2: Phonology. Stanford: Stanford University Press, 1978.

Crystal, David. Diccionario de lingüistica y Fonética. Barcelona: Octaedro, 2000.

Hooper, Joan B. An Introduction to Natural Generative Phonology. Nueva York: Academic Press, 1976.

Jakobson, Roman y Linda Waugh. La forma sonora de la lengua. México: Fondo de Cultura Económica, 1987.

Obediente, Enrique. Fonética y Fonología. Mérida: Consejo de Publicaciones de la Universidad de los Andes, 2007.

Martínez-Celdrán, Eugenio. El sonido en la comunicación humana. Barcelona: Octaedro, 1996.

Fonética, Barcelona: Teide, 1993. $4^{\mathrm{a}}$ edición revisada.

Quilis, Antonio y Joseph Fernández. Curso de fonética y fonología españolas: para estudiantes angloamericanos. Madrid: Consejo Superior de Investigaciones Científicas, 1973.

Real Academia Española. Diccionario de la Lengua Española. Utilizamos versión on line disponible en http://www.rae.es/rae.html.

Samarin, Williams. Tongues of Men and Angels. New York: Macmillan, 1972.

- "Variation and variables in Religious Glossolalia". Language in Society 1, Cambridge: Cambridge University Press, 1972, Pág. 121-130.

Trubetzkoy, Nicolai. Principios de Fonología. Madrid: Cincel, 1969[1939].

\section{ANEXOS}

4.1 Transcripciones fonéticas y estructuras silábicas ${ }^{6}$

Transcripción fonética de enunciados de D. G.

1. [:o $\left.]_{v} . \delta o .: \rho 0 . \pi \alpha . \varphi \alpha .: \sigma \imath . \alpha .: \mu \alpha . \eta \alpha: \Sigma 1 . \alpha \subseteq\right]$

1. $\mathrm{VC}, \mathrm{CV}, \mathrm{CV}, \mathrm{CV}, \mathrm{CV}, \mathrm{CV}, \mathrm{V}, \mathrm{CV}, \mathrm{CV}, \mathrm{CV}, \mathrm{V}$

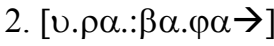

2. $\mathrm{V}, \mathrm{CV}, \mathrm{CV}, \mathrm{CV}$

\footnotetext{
${ }^{6}$ Conviene señalar que la mayoría de los enunciados que siguen corresponden a grupos fónicos pronunciados por los sujetos en distintos momentos de sus sermones, por lo que, en estricto rigor, no forman parte de un mismo "texto".
} 


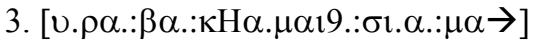

3. $\mathrm{V}, \mathrm{CV}, \mathrm{CV}, \mathrm{CV}, \mathrm{CD}, \mathrm{CV}, \mathrm{V}, \mathrm{CV}$

4. [v. $\rho \alpha .: \beta \alpha .: \kappa \alpha . \mu \alpha . \varphi \alpha .: \sigma 1 . \alpha \rightarrow]$

4. $\mathrm{V}, \mathrm{CV}, \mathrm{CV}, \mathrm{CV}, \mathrm{CV}, \mathrm{CV}, \mathrm{CV}, \mathrm{V}$

5. [: $\alpha 19 . \sigma \alpha .: \mu \alpha . \times \alpha \rightarrow]$

5. D, CV, CV, CV

6. [: $\left.\mu \alpha 19 . \tau^{\sigma} 1 .: \Delta 4 \alpha . \mu \alpha .: \kappa H \alpha . \mu \alpha .: \sigma o . \varphi \alpha \rightarrow\right]$

6. $\mathrm{CD}, \mathrm{CV}, \mathrm{CV}, \mathrm{CV}, \mathrm{CV}, \mathrm{CV}, \mathrm{CV}, \mathrm{CV}$

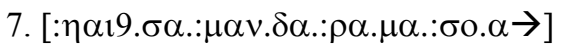

7. $\mathrm{CD}, \mathrm{CV}, \mathrm{CVC}, \mathrm{CV}, \mathrm{CV}, \mathrm{CV}, \mathrm{CV}, \mathrm{V}$

$8[: \eta \alpha 19 . \sigma \alpha .: \mu \alpha v . \delta \alpha .: \delta \alpha . \mu \alpha .: 1 . \alpha . \sigma o\rceil \rightarrow]$

$8 \mathrm{CD}, \mathrm{CV}, \mathrm{CVC}, \mathrm{CV}, \mathrm{CV}, \mathrm{CV}, \mathrm{CV}, \mathrm{V}, \mathrm{V}, \mathrm{CV}$

$9[: \cup . \rho \alpha: \pi \alpha .: \pi \alpha .: \pi \alpha .: \pi \alpha .: \pi \alpha .: \pi \alpha .: \pi \alpha . \pi \alpha 19 .: \sigma \imath . \alpha \subseteq]$

$9 \mathrm{~V}, \mathrm{CV}, \mathrm{CV}, \mathrm{CV}, \mathrm{CV}, \mathrm{CV}, \mathrm{CV}, \mathrm{CV}, \mathrm{CV}, \mathrm{CD}, \mathrm{CV}, \mathrm{V}$

$10[: v . \sigma \alpha .: \delta \alpha . \times \alpha .: \mu \alpha \mu . \beta 1 .: \mathrm{P} \imath . \sigma o \rightarrow]$

$10 \mathrm{~V}, \mathrm{CV}, \mathrm{CV}, \mathrm{CV}, \mathrm{CVC}, \mathrm{CV}, \mathrm{CV}, \mathrm{CV}$

$11[: \alpha \nu . \delta \alpha . \lambda \alpha . \beta \alpha 19 .: \sigma \varepsilon . \Delta 4 \varepsilon \rightarrow]$

$11 \mathrm{VC}, \mathrm{CV}, \mathrm{CV}, \mathrm{CD}, \mathrm{CV}, \mathrm{CV}$

$\left.12[: \alpha 19 . \sigma \alpha . \cup \mu \alpha\rceil v . \delta \alpha . \lambda \alpha . \varpi \alpha 19 .: \sigma o\rceil v . \delta o . \lambda \circ . \mu \alpha v .: \delta \varepsilon . P \varepsilon . \varpi \varepsilon .: \sigma \varepsilon\rceil_{1} 9 . \sigma \alpha \rightarrow\right]$

$12 \mathrm{D}, \mathrm{CV}, \mathrm{CVC}, \mathrm{CV}, \mathrm{CV}, \mathrm{CD}, \mathrm{CVC}, \mathrm{CV}, \mathrm{CV}, \mathrm{CVC}, \mathrm{CV}, \mathrm{CV}, \mathrm{CV}, \mathrm{CD}, \mathrm{CV}$

$13[\kappa H \alpha .: \mu \alpha v . \delta \alpha . \lambda \alpha . \beta \alpha 19 .: \sigma \sigma . \delta o . \lambda o .: \beta \alpha 19 \sigma \subset]$

$13 \mathrm{CV}, \mathrm{CVC}, \mathrm{CV}, \mathrm{CV}, \mathrm{CD}, \mathrm{CV}, \mathrm{CV}, \mathrm{CV}, \mathrm{CDC}$

$\left.14[: \eta \alpha 19 . \sigma \alpha .: \mu \alpha v . \delta \alpha . \rho \alpha . \beta \alpha .: \sigma o]_{v} . \delta o . \lambda o .: \pi \alpha .: \pi \alpha .: \pi \alpha . \varphi \alpha .: \sigma 1 . \alpha \rightarrow\right]$

$14 \mathrm{CD}, \mathrm{CV}, \mathrm{CVC}, \mathrm{CV}, \mathrm{CV}, \mathrm{CV}, \mathrm{CVC}, \mathrm{CV}, \mathrm{CV}, \mathrm{CV}, \mathrm{CV}, \mathrm{CV}, \mathrm{CV}, \mathrm{CV}, \mathrm{V}$

$15[: \Sigma \alpha .: \kappa 1 . \alpha .: \mu \alpha v \rightarrow]$

$15 \mathrm{CV}, \mathrm{CV}, \mathrm{V}, \mathrm{CVC}$

Transcripción fonética de enunciados de Y. Á.

\section{Las Diez Vírgenes}

$1[: \Sigma \alpha . \mu \alpha .: \eta \alpha 19 \rightarrow]$

$1 \mathrm{CV}, \mathrm{CV}, \mathrm{CD}$ 
$2[: \alpha 19 . \sigma \alpha .: \lambda 1 . \alpha .: \eta \alpha .: \eta \alpha . \varphi \alpha .: \sigma o . \alpha .: \mu \alpha \rightarrow]$

$2 \mathrm{D}, \mathrm{CV}, \mathrm{CV}, \mathrm{V}, \mathrm{CV}, \mathrm{CV}, \mathrm{CV}, \mathrm{CV}, \mathrm{V}, \mathrm{CV}$

$3[: \Sigma o . \beta \alpha .: \eta \alpha . \varphi \alpha .: \mu \alpha \rightarrow]$

$3 \mathrm{CV}, \mathrm{CV}, \mathrm{CV}, \mathrm{CV}, \mathrm{CV}$

$4[\mu \alpha .: \eta \alpha 19 . \lambda \imath . \alpha: \sigma o . \eta \alpha .: \eta \alpha . \lambda 1 . \alpha . \beta \alpha \rightarrow]$

$4 \mathrm{CV}, \mathrm{CD}, \mathrm{CV}, \mathrm{V}, \mathrm{CV}, \mathrm{CV}, \mathrm{CV}, \mathrm{CV}, \mathrm{V}, \mathrm{CV}$

\section{La Desobediencia}

$1[: \alpha 19 . \sigma \alpha .: \mu \alpha . \varphi \alpha .: \sigma o . \eta \alpha .: \varpi \alpha] \subseteq]$

$1 \mathrm{D}, \mathrm{CV}, \mathrm{CV}, \mathrm{CV}, \mathrm{CV}, \mathrm{CV}, \mathrm{CV}$ :

$2[: \alpha \sigma .: \sigma \alpha . \mu \alpha . \varphi \alpha .: \sigma o . \eta \alpha \rightarrow]$

$2 \mathrm{VC}, \mathrm{CV}, \mathrm{CV}, \mathrm{CV}, \mathrm{CV}, \mathrm{CV}$

$3[: \alpha . \varphi \alpha .: \sigma o . \eta \alpha .: \mu \alpha \subseteq]$

$3 \mathrm{~V}, \mathrm{CV}, \mathrm{CV}, \mathrm{CV}, \mathrm{CV}$

$4[: \alpha 19 . \sigma \alpha .: \mu \alpha . \varphi \alpha .: \sigma o . \eta \alpha . \varpi \alpha\rceil \rightarrow]$

$4 \mathrm{D}, \mathrm{CV}, \mathrm{CV}, \mathrm{CV}, \mathrm{CV}, \mathrm{CV}, \mathrm{CV}$ :

$5[: \alpha 19 .: \sigma \alpha . \mu \alpha . \varphi \alpha .: \sigma o . \eta \alpha . \varpi \alpha \subseteq]$

$5 \mathrm{D}, \mathrm{CV}, \mathrm{CV}, \mathrm{CV}, \mathrm{CV}, \mathrm{CV}, \mathrm{CV}$

$6[: \mu \alpha . \varphi \alpha .: \sigma o . \eta \alpha . \mu \alpha \subseteq]$

$6 \mathrm{CV}, \mathrm{CV}, \mathrm{CV}, \mathrm{CV}, \mathrm{CV}$

$7[: \alpha . \sigma \alpha . \mu \alpha . \varphi \alpha .: \sigma o . \eta \alpha\rceil \rightarrow]$

$7 \mathrm{~V}, \mathrm{CV}, \mathrm{CV}, \mathrm{CV}, \mathrm{CV}, \mathrm{CV}$ : 


\section{El Rapto}

$1[: \alpha 19 . \lambda \alpha .: \sigma \alpha . \eta \alpha . \mu \alpha . \varphi \alpha .: \sigma o\rceil . \eta \alpha \rightarrow]$

$1 \mathrm{D}, \mathrm{CV}, \mathrm{CV}, \mathrm{CV}, \mathrm{CV}, \mathrm{CV}, \mathrm{CV}: \mathrm{CV}$

$2[: \alpha 19 . \lambda \alpha .: \sigma \alpha . \eta \alpha .: \mu \alpha\rceil \rightarrow]$

$2 \mathrm{D}, \mathrm{CV}, \mathrm{CV}, \mathrm{CV}, \mathrm{CV}$ :

$3[: \alpha 19 . \sigma \alpha . \mu \alpha .: \sigma o . \eta \alpha\rceil \rightarrow]$

$3 \mathrm{D}, \mathrm{CV}, \mathrm{CV}, \mathrm{CV}, \mathrm{CV}$ :

$4[: \alpha 19 . \sigma \alpha .: \eta \alpha . \mu \alpha .: \lambda o . \alpha\rceil \rightarrow]$

$4 \mathrm{D}, \mathrm{CV}, \mathrm{CV}, \mathrm{CV}, \mathrm{CV}, \mathrm{V}$ :

$5[: \alpha 19 .: \sigma \alpha\rceil . \lambda \alpha . \mu \alpha 19 \rightarrow]$

$5 \mathrm{D}, \mathrm{CV}: \mathrm{CV}, \mathrm{CD}$

$6[: \alpha 19 .: \lambda \alpha . \sigma \alpha . \mu \alpha .: \eta \alpha . \varphi \alpha .: \sigma o . \beta \alpha\rceil \rightarrow]$

$6 \mathrm{D}, \mathrm{CV}, \mathrm{CV}, \mathrm{CV}, \mathrm{CV}, \mathrm{CV}, \mathrm{CV}, \mathrm{CV}$ :

$7[: \alpha 19 . \sigma \alpha .: \eta \alpha . \mu \alpha .: \lambda \circ . \eta \alpha\rceil \rightarrow]$

$7 \mathrm{D}, \mathrm{CV}, \mathrm{CV}, \mathrm{CV}, \mathrm{CV}, \mathrm{CV}$ :

$8[: \alpha 19 . \sigma \alpha .: \eta \alpha . \mu \alpha .: \lambda \circ . \eta \alpha .: \eta \alpha] \subseteq]$

$8 \mathrm{D}, \mathrm{CV}, \mathrm{CV}, \mathrm{CV}, \mathrm{CV}, \mathrm{CV}, \mathrm{CV}$ :

Transcripción fonética de enunciados de E. B.

$1[\alpha .: \rho \alpha .: \beta \alpha .: \beta \alpha .: \beta \alpha .: B 4 \alpha .: B 4 \alpha .: \Sigma \alpha v . \delta \alpha . P \alpha . P \alpha .: B 4 \alpha .: B 4 \alpha .: \tau \varepsilon . \varpi \alpha \rightarrow]$

$1 \mathrm{~V}, \mathrm{CV}, \mathrm{CV}, \mathrm{CV}, \mathrm{CV}, \mathrm{CV}, \mathrm{CV}, \mathrm{CVC}, \mathrm{CV}, \mathrm{CV}, \mathrm{CV}, \mathrm{CV}, \mathrm{CV}, \mathrm{CV}, \mathrm{CV}$

$2[: \sigma\rceil \alpha . \varphi \alpha . \mu \alpha . \rho \alpha .: B 4 \alpha .: \kappa о . \tau о .: \rho о .: \beta о .: \Sigma \varepsilon . \tau \varepsilon .: \rho \varepsilon .: B 4 \varepsilon . \tau \imath . P \imath . \varpi \alpha: . \sigma \varepsilon . \kappa \alpha .: \tau \alpha . P \alpha \rightarrow]$

$2 \mathrm{CV}, \mathrm{CV}, \mathrm{CV}, \mathrm{CV}, \mathrm{CV}, \mathrm{CV}, \mathrm{CV}, \mathrm{CV}, \mathrm{CV}, \mathrm{CV}, \mathrm{CV}, \mathrm{CV}, \mathrm{CV}, \mathrm{CV}, \mathrm{CV}, \mathrm{CV}, \mathrm{CV}, \mathrm{CV}, \mathrm{CV}$, $\mathrm{CV}$

$3[0 .: \rho \varepsilon .: \beta \varepsilon .: \beta \varepsilon .: \Sigma 1 . \kappa \alpha .: \rho \alpha . \beta \alpha .: \eta \alpha 19 \rightarrow]$

$3 \mathrm{~V}, \mathrm{CV}, \mathrm{CV}, \mathrm{CV}, \mathrm{CV}, \mathrm{CV}, \mathrm{CV}, \mathrm{CV}, \mathrm{CD}$

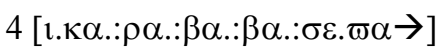

$4 \mathrm{~V}, \mathrm{CV}, \mathrm{CV}, \mathrm{CV}, \mathrm{CV}, \mathrm{CV}, \mathrm{CV}$

5 [о. $\alpha$. $\beta \alpha .: \Sigma \varepsilon v . \delta \varepsilon . P \varepsilon . P \varepsilon . \beta \varepsilon .: \kappa \iota . P \imath . \beta \alpha: \sigma \leftrightarrow . \tau \alpha . P \alpha .: \pi \alpha . \pi \alpha .: \eta \mathrm{o}\rceil]]$

$5 \mathrm{~V}, \mathrm{CV}, \mathrm{CV}, \mathrm{CVC}, \mathrm{CV}, \mathrm{CV}, \mathrm{CV}, \mathrm{CV}, \mathrm{CV}, \mathrm{CV}, \mathrm{CV}, \mathrm{CV}, \mathrm{CV}, \mathrm{CV}, \mathrm{CV}, \mathrm{CV}, \mathrm{CV}$ :

6 [:о.: $\rho 1 . \beta \alpha . \beta \alpha .: \Sigma \varepsilon v . \delta \alpha .: P \alpha . \lambda \alpha . \beta \alpha . \kappa P \imath . \beta \alpha . \sigma \varepsilon . \tau \varepsilon . P \varepsilon . \varphi \alpha .: \mu \alpha v . \delta \alpha .: P \alpha . \beta \alpha .: \Sigma 1 . \sigma \alpha \rightarrow]$ 
$6 \mathrm{~V}, \mathrm{CV}, \mathrm{CV}, \mathrm{CV}, \mathrm{CVC}, \mathrm{CV}, \mathrm{CV}, \mathrm{CV}, \mathrm{CV}, \mathrm{CCV}, \mathrm{CV}, \mathrm{CV}, \mathrm{CV}, \mathrm{CV}, \mathrm{CV}, \mathrm{CVC}, \mathrm{CV}, \mathrm{CV}$, $\mathrm{CV}, \mathrm{CV}, \mathrm{CV}$

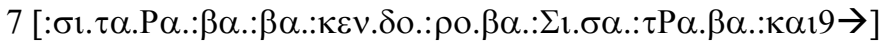

$7 \mathrm{CV}, \mathrm{CV}, \mathrm{CV}, \mathrm{CV}, \mathrm{CV}, \mathrm{CVC}, \mathrm{CV}, \mathrm{CV}, \mathrm{CV}, \mathrm{CV}, \mathrm{CV}, \mathrm{CCV}, \mathrm{CV}, \mathrm{CD}$

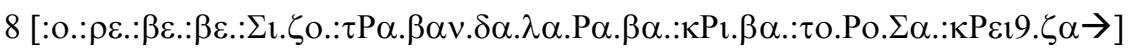

$8 \mathrm{~V}, \mathrm{CV}, \mathrm{CV}, \mathrm{CV}, \mathrm{CV}, \mathrm{CV}, \mathrm{CCV}, \mathrm{CVC}, \mathrm{CV}, \mathrm{CV}, \mathrm{CV}, \mathrm{CV}, \mathrm{CCV}, \mathrm{CV}, \mathrm{CV}, \mathrm{CV}, \mathrm{CV}, \mathrm{CCD}$, $\mathrm{CV}$

$9[\tau \varepsilon . \rho \varepsilon . \beta \varepsilon .: \tau v . \rho 1 . \alpha .: \kappa \alpha v . \delta \alpha .: \rho \alpha . \beta \alpha .: \Sigma \varepsilon .: \kappa P o v . \delta \alpha .: \Delta 4 \alpha . \beta \alpha .: \tau$ P $\varepsilon \sigma . \tau 0 .: \kappa P \alpha . \mu \alpha .: \tau \varepsilon 19 . \beta \alpha$ $\sigma .: \sigma \mathrm{l} .: \tau 0 . \lambda \mathrm{o} . \beta \alpha .: \Sigma \varepsilon 19 \sigma \rightarrow]$

$9 \mathrm{CV}, \mathrm{CV}, \mathrm{CV}, \mathrm{CV}, \mathrm{CV}, \mathrm{V}, \mathrm{CVC}, \mathrm{CV}, \mathrm{CV}, \mathrm{CV}, \mathrm{CV}, \mathrm{CCVC}, \mathrm{CV}, \mathrm{CV}, \mathrm{CV}, \mathrm{CCVC}, \mathrm{CV}$, $\mathrm{CCV}, \mathrm{CV}, \mathrm{CD}, \mathrm{CVC}, \mathrm{CV}, \mathrm{CV}, \mathrm{CV}, \mathrm{CV}, \mathrm{CDC}$

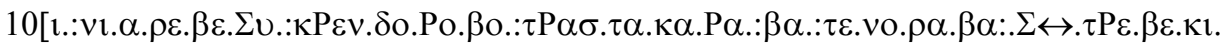
$v \alpha .: \lambda \alpha 19 \sigma . \sigma o .: \kappa$ Po. $\Sigma \alpha .: v \alpha . v \alpha .: \sigma \varepsilon 19 \sigma \subseteq]$

$10 \mathrm{~V}, \mathrm{CV}, \mathrm{V}, \mathrm{CV}, \mathrm{CV}, \mathrm{CV}, \mathrm{CCVC}, \mathrm{CV}, \mathrm{CV}, \mathrm{CV}, \mathrm{CCVC}, \mathrm{CV}, \mathrm{CV}, \mathrm{CV}, \mathrm{CV}, \mathrm{CV}, \mathrm{CV}, \mathrm{CV}$, $\mathrm{CV}, \mathrm{CV}, \mathrm{CCV}, \mathrm{CV}, \mathrm{CV}, \mathrm{CV}, \mathrm{CDC}, \mathrm{CV}, \mathrm{CCV}, \mathrm{CV}, \mathrm{CV}, \mathrm{CV}, \mathrm{CDC}$

11

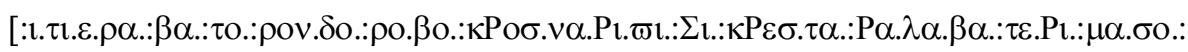
ко. $\alpha \alpha \rightarrow]$

$11 \mathrm{~V}, \mathrm{CV}, \mathrm{V}, \mathrm{CV}, \mathrm{CV}, \mathrm{CV}, \mathrm{CVC}, \mathrm{CV}, \mathrm{CV}, \mathrm{CV}, \mathrm{CCVC}, \mathrm{CV}, \mathrm{CV}, \mathrm{CV}, \mathrm{CV}, \mathrm{CCVC}, \mathrm{CV}, \mathrm{CV}$, $\mathrm{CV}, \mathrm{CV}, \mathrm{CV}, \mathrm{CV}, \mathrm{CV}, \mathrm{CV}, \mathrm{CV}, \mathrm{CV}$

$12[: 1 .: \mu 1 . \alpha .:$ бо.ко.ро. $\beta \alpha .: \Sigma \varepsilon v . \delta v .: \rho v . \beta \alpha . \tau \alpha \imath 9 \subset]$

$12 \mathrm{~V}, \mathrm{CV}, \mathrm{V}, \mathrm{CV}, \mathrm{CV}, \mathrm{CV}, \mathrm{CV}, \mathrm{CVC}, \mathrm{CV}, \mathrm{CV}, \mathrm{CV}, \mathrm{CD}$

$13[: \sigma o . \alpha .: \tau \varepsilon . P \varepsilon .: \mu \alpha v . \delta o .: \beta o . \varphi \alpha .: \Sigma \varepsilon .: \kappa \varepsilon . P \varepsilon . \mu \alpha .: \sigma \alpha v . \delta \alpha \subseteq]$

$13 \mathrm{CV}, \mathrm{V}, \mathrm{CV}, \mathrm{CV}, \mathrm{CVC}, \mathrm{CV}, \mathrm{CV}, \mathrm{CV}, \mathrm{CV}, \mathrm{CV}, \mathrm{CV}, \mathrm{CV}, \mathrm{CVC}, \mathrm{CV}$

$14[: \varepsilon \sigma . \tau \alpha .: \kappa P 1 . \mu v .: \Sigma v . \kappa \alpha . P \alpha v . \delta o .: \beta o .: \Sigma o .: \kappa P \varepsilon 19 . \tau \alpha \rightarrow]$

$14 \mathrm{VC}, \mathrm{CV}, \mathrm{CCV}, \mathrm{CV}, \mathrm{CV}, \mathrm{CV}, \mathrm{CVC}, \mathrm{CV}, \mathrm{CV}, \mathrm{CV}, \mathrm{CCD}, \mathrm{CV}$

15

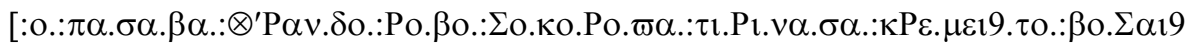
$\zeta \subseteq]$

$15 \mathrm{~V}, \mathrm{CV}, \mathrm{CV}, \mathrm{CV}, \mathrm{CCVC}, \mathrm{CV}, \mathrm{CV}, \mathrm{CV}, \mathrm{CV}, \mathrm{CV}, \mathrm{CV}, \mathrm{CV}, \mathrm{CV}, \mathrm{CV}, \mathrm{CV}, \mathrm{CV}, \mathrm{CCV}, \mathrm{CD}, \mathrm{CV}$, $\mathrm{CV}, \mathrm{CDC}$

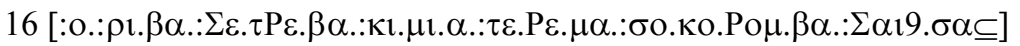

$16 \mathrm{~V}, \mathrm{CV}, \mathrm{CV}, \mathrm{CV}, \mathrm{CCV}, \mathrm{CV}, \mathrm{CV}, \mathrm{CV}, \mathrm{V}, \mathrm{CV}, \mathrm{CV}, \mathrm{CV}, \mathrm{CV}, \mathrm{CV}, \mathrm{CVC}, \mathrm{CV}, \mathrm{CD}, \mathrm{CV}$ 
17

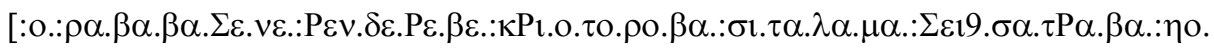
$\varpi \alpha \rightarrow]$

$17 \mathrm{~V}, \mathrm{CV}, \mathrm{CV}, \mathrm{CV}, \mathrm{CV}, \mathrm{CV}, \mathrm{CVC}, \mathrm{CV}, \mathrm{CV}, \mathrm{CV}, \mathrm{CCV}, \mathrm{V}, \mathrm{CV}, \mathrm{CV}, \mathrm{CV}, \mathrm{CV}, \mathrm{CV}, \mathrm{CV}, \mathrm{CV}$, $\mathrm{CVV}, \mathrm{CV}, \mathrm{CCV}, \mathrm{CV}, \mathrm{CV}, \mathrm{CV}$

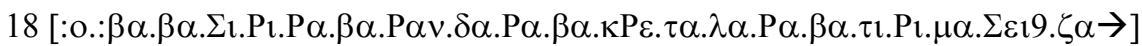

$18 \mathrm{~V}, \mathrm{CV}, \mathrm{CV}, \mathrm{CV}, \mathrm{CV}, \mathrm{CV}, \mathrm{CV}, \mathrm{CVC}, \mathrm{CV}, \mathrm{CV}, \mathrm{CV}, \mathrm{CCV}, \mathrm{CV}, \mathrm{CV}, \mathrm{CV}, \mathrm{CV}, \mathrm{CV}, \mathrm{CV}, \mathrm{CV}$, $\mathrm{CD}, \mathrm{CV}$

$19[: 1 . \sigma \alpha .: \rho \alpha . B 4 \alpha .: \Sigma \varepsilon . P \varepsilon .: P \varepsilon v . \delta \alpha .: \lambda \alpha . \varpi \alpha .: 1 . \alpha: \tau о . \rho \alpha .: \mu \alpha . \sigma o .: \eta о . \varpi \alpha \rightarrow]$

$19 \mathrm{~V}, \mathrm{CV}, \mathrm{CV}, \mathrm{CV}, \mathrm{CV}, \mathrm{CV}, \mathrm{CVC}, \mathrm{CV}, \mathrm{CV}, \mathrm{CV}, \mathrm{V}, \mathrm{V}, \mathrm{CV}, \mathrm{CV}, \mathrm{CV}, \mathrm{CV}, \mathrm{CV}, \mathrm{CV}$

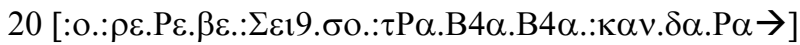

$20 \mathrm{~V}, \mathrm{CV}, \mathrm{CV}, \mathrm{CV}, \mathrm{CD}, \mathrm{CV}, \mathrm{CCV}, \mathrm{CV}, \mathrm{CV}, \mathrm{CVC}, \mathrm{CV}, \mathrm{CV}$

$21\left[:\right.$ o.: $\pi \varepsilon$. Po.ı. $\left.\mu \alpha . \sigma \alpha . \kappa P \varepsilon . \alpha . B 4 o .: \Sigma_{1} \rightarrow\right]$

$21 \mathrm{~V}, \mathrm{CV}, \mathrm{CV}, \mathrm{V}, \mathrm{CV}, \mathrm{CV}, \mathrm{CCV}, \mathrm{V}, \mathrm{CV}, \mathrm{CV}$

$22[: \mathrm{o}] .: \rho \varepsilon . \mathrm{B} 4 \varepsilon . \mathrm{B} 4 \varepsilon .: \Sigma \varepsilon 19 . \sigma \mathrm{\sigma o} .: \tau \mathrm{P} \alpha . \mathrm{B} 4 \alpha . \mathrm{B} 4 \alpha .: \eta \alpha 19 \rightarrow]$

$22 \mathrm{~V}\rceil, \mathrm{CV}, \mathrm{CV}, \mathrm{CD}, \mathrm{CV}, \mathrm{CCV}, \mathrm{CV}, \mathrm{CV}, \mathrm{CD}$

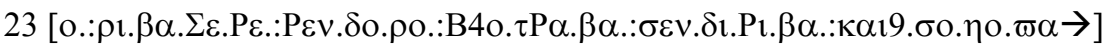

$23 \mathrm{~V}, \mathrm{CV}, \mathrm{CV}, \mathrm{CV}, \mathrm{CV}, \mathrm{CVC}, \mathrm{CV}, \mathrm{CV}, \mathrm{CV}, \mathrm{CCV}, \mathrm{CV}, \mathrm{CVC}, \mathrm{CV}, \mathrm{CV}, \mathrm{CV}, \mathrm{CD}, \mathrm{CV}, \mathrm{CV}, \mathrm{CV}$

24 [o. $\beta \alpha . \Sigma \alpha .: P \alpha v . \delta \alpha . P \alpha . B 4 \alpha .: \kappa P ı . \sigma o .: \delta 1 . \alpha . \beta \alpha .: \tau \alpha v . \delta o . \rho o . \beta \alpha .: \Sigma \varepsilon 19 \zeta \rightarrow]$

$24 \mathrm{~V}, \mathrm{CV}, \mathrm{CV}, \mathrm{CVC}, \mathrm{CV}, \mathrm{CV}, \mathrm{CV}, \mathrm{CCV}, \mathrm{CV}, \mathrm{CV}, \mathrm{V}, \mathrm{CV}, \mathrm{CVC}, \mathrm{CV}, \mathrm{CV}, \mathrm{CV}, \mathrm{CDC}$ 